\title{
Structural evolution in liquid Galn eutectic alloy under high temperature and pressure
}

Yu, Q.; Su, Y.; Wang, X. D.; Ståhl, Kenny; Glazyrin, K.; Liermann, H. P.; Franz, H.; Cao, Q. P.; Zhang, D. X.; Jiang, J. Z.

Published in:

Journal of Applied Physics

Link to article, DOI:

$10.1063 / 1.5098036$

Publication date:

2019

Document Version

Publisher's PDF, also known as Version of record

Link back to DTU Orbit

Citation (APA):

Yu, Q., Su, Y., Wang, X. D., Ståhl, K., Glazyrin, K., Liermann, H. P., Franz, H., Cao, Q. P., Zhang, D. X., \& Jiang, J. Z. (2019). Structural evolution in liquid Galn eutectic alloy under high temperature and pressure. Journal of Applied Physics, 126(1), [015902]. https://doi.org/10.1063/1.5098036

\section{General rights}

Copyright and moral rights for the publications made accessible in the public portal are retained by the authors and/or other copyright owners and it is a condition of accessing publications that users recognise and abide by the legal requirements associated with these rights.

- Users may download and print one copy of any publication from the public portal for the purpose of private study or research.

- You may not further distribute the material or use it for any profit-making activity or commercial gain

- You may freely distribute the URL identifying the publication in the public portal 


\section{Structural evolution in liquid Galn eutectic alloy under high temperature and pressure}

Cite as: J. Appl. Phys. 126, 015902 (2019); https://doi.org/10.1063/1.5098036

Submitted: 31 March 2019 . Accepted: 15 June 2019 . Published Online: 01 July 2019

Q. Yu (D) Y. Su, X. D. Wang (D), K. Ståhl, K. Glazyrin (D), H. P. Liermann (D), H. Franz, Q. P. Cao, D. X. Zhang, and J. Z. Jiang (D)

\section{rA AlluXa rouroptical COATING PARTNER}




\title{
Structural evolution in liquid Galn eutectic alloy under high temperature and pressure
}

\author{
Cite as: J. Appl. Phys. 126, 015902 (2019); doi: 10.1063/1.5098036 \\ Submitted: 31 March 2019 . Accepted: 15 June 2019 . \\ Published Online: 1 July 2019
}

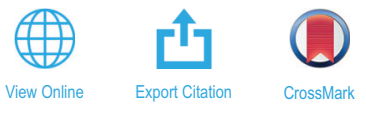

Q. Yu, ${ }^{1}$ (D) Y. Su, ${ }^{7}$ X. D. Wang, ${ }^{1, a)}$ (D) K. Ståhl, ${ }^{2}$ K. Glazyrin, ${ }^{3}$ (D) H. P. Liermann, ${ }^{3}$ (D) H. Franz, ${ }^{3}$ Q. P. Cao, ${ }^{1}$ D. X. Zhang, ${ }^{1,4}$ and J. Z. Jiang ${ }^{1, a)}$ (iD

\author{
AFFILIATIONS \\ ${ }^{1}$ International Center for New-Structured Materials (ICNSM), Laboratory of New-Structured Materials, State Key Laboratory of Silicon \\ Materials, and School of Materials Science and Engineering, Zhejiang University, Hangzhou 310027, People's Republic of China \\ ${ }^{2}$ Department of Chemistry, Technical University of Denmark, DK-2800 Lyngby, Denmark \\ ${ }^{3}$ DESY, Photon Science, D-22603 Hamburg, Germany \\ ${ }^{4}$ State Key Laboratory of Modern Optical Instrumentation, Zhejiang University, Hangzhou 310027, People's Republic of China
}

${ }^{a)}$ Authors to whom correspondence should be addressed: jiangjz@zju.edu.cn and wangxd@zju.edu.ch

\begin{abstract}
The structural evolution of a liquid GaIn eutectic alloy under high temperature and high pressure is investigated by combining in situ X-ray diffraction (XRD) and $a b$ initio molecular dynamics simulations. Both experimental and theoretical results confirm that no pressureinduced sudden structural changes are detected in the liquid state along different isotherms below $700 \mathrm{~K}$. The XRD patterns indicate that the liquids at 400 and $673 \mathrm{~K}$ both crystallize into a tetragonal crystalline phase under high pressure, whose structure is locally face centered cubic ( $\mathrm{fcc}$ )-like. The theoretical simulations successfully describe the atomic-scale structural evolution from disordered liquid to ordered solid phases during the isothermal compression at different temperatures, revealing a strong competition between the body-centered cubic (bcc)-like and fcc-like local atomic packings at the early stage of nucleation. The liquid can directly solidify into the bcc-like atomic packing at temperatures above $650 \mathrm{~K}$, whereas this bcc-like structure becomes transient and metastable below $600 \mathrm{~K}$ and finally transforms into a stable fcc-like atomic packing with increasing pressure. Furthermore, a high-pressure and high-temperature "phase diagram" of the GaIn eutectic alloy is roughly constructed, providing new insight into atomic-scale disorder-to-order transition of the liquid GaIn eutectic alloy in extreme conditions.
\end{abstract}

Published under license by AIP Publishing. https://doi.org/10.1063/1.5098036

\section{INTRODUCTION}

Phase transitions in metallic liquids, including crystallization, vitrification, and liquid-to-liquid transition, have attracted considerable attention due to their key roles in industrial manufacturing and fundamental science. ${ }^{1-8}$ Particularly, the crystallization process is crucial in controlling as-solidified microstructures of products. Although the observation of crystallization in metallic liquids at the atomic scale is still challenging, developments in experimental characterizations and theoretical simulations have greatly improved the understanding of crystallization processes of metallic liquids. ${ }^{7-13}$ Due to nontoxicity, superior electrical conductivity, low viscosity, and perfect deformability, eutectic GaIn and GaIn-based alloys have gained increasing popularity in flexible and stretchable electronics applications. ${ }^{14-16}$ Here, we select a binary $\mathrm{Ga}_{85.8} \mathrm{In}_{14.2}$ eutectic alloy (at. \%) as a model system to investigate the microstructure evolution in a broad range of temperatures and pressures due to its low melting point $\left(\sim 15^{\circ} \mathrm{C}\right)$. Our previous studies ${ }^{17}$ revealed that an unusual temperature-induced liquid-to-liquid crossover appears at $400-550 \mathrm{~K}$ at ambient pressure in the GaIn eutectic alloy. When pressure was applied to the liquid sample at room temperature, no abnormal structural change was detected prior to solidification. The liquid can directly solidify into a monoclinic phase at about $3.4 \mathrm{GPa}$ and further increasing the pressure resulted in the polymorphic transition from monoclinic to triclinic structures at $10.3 \mathrm{GPa} .{ }^{18}$ Hence, some questions emerge: Is there a pressure-induced abnormal behavior in the high-temperature liquid state? How does the liquid structure change in high-pressure and high-temperature conditions? Is there any difference in pressure-induced solidification processes for the low- and high-temperature liquids? To answer these questions, 
we performed in situ X-ray diffraction (XRD) experiments for the liquid GaIn eutectic alloy under high pressure $(P)$ and temperature (T) up to $32.6 \mathrm{GPa}$ and $673 \mathrm{~K}$ using resistance-heated diamond anvil cells (DACs) together with series of $a b$ initio molecular dynamics (AIMD) calculations at pressures up to about $80 \mathrm{GPa}$ and temperatures up to $700 \mathrm{~K}$. The structural evolution in liquid states and the subsequent solidification process are studied under various pressure and temperature conditions.

\section{EXPERIMENTAL AND THEORETICAL METHODS}

\section{A. In situ high-pressure and high-temperature (high $P-T$ ) $\mathrm{X}$-ray diffraction}

Isothermal high-pressure XRD experiments at $400 \mathrm{~K}$ and $673 \mathrm{~K}$ were performed using internal resistance-heated diamond anvil cells (DACs) equipped with a $300 \mu \mathrm{m}$ diameter culet. A $120 \mu \mathrm{m}$ hole was drilled as a sample chamber in a Re gasket. The liquid GaIn eutectic alloy prepared with high purity Ga (99.99\%) and In (99.99\%) was injected into the sample chamber without a pressure transmitting medium. A small piece of ruby or tungsten was embedded in the sample at $400 \mathrm{~K}$ and $673 \mathrm{~K}$, respectively, to determine the pressure inside the DAC by the standard ruby fluorescence and the $P-V$ equation of state of tungsten (W). ${ }^{19}$ To our knowledge, there is no W-Ga phase diagram published until now. At very high temperature $(673 \mathrm{~K})$ and pressure $(7.7 \mathrm{GPa})$, a metastable intermetallic phase $\mathrm{W}_{2} \mathrm{Ga}_{5}\left(\mathrm{Mn}_{2} \mathrm{Hg}_{5}\right.$ structure) and a solid solution of $\mathrm{Ga}$ in $\mathrm{W}$ (bcc) have been prepared by Popova and Fomicheva. ${ }^{20}$ Here, no detectable diffraction peaks except pure $\mathrm{W}$ was observed; thus, we assumed that no reaction occurs between $\mathrm{Ga}$ and $\mathrm{W}$ during our experiments. Prior to the experiment, the sample chamber was evacuated down to about $10^{-4}$ mbar. The sample was then heated to the desired temperatures and held for $15 \mathrm{~min}$ to ensure temperature stability. The sample temperature was determined from a thermocouple placed directly on one of the anvils very closely to the sample chamber. During experiments, the temperature almost remained stable within the experimental uncertainty of $0.2 \mathrm{~K}$. The high $P-T$ XRD patterns were measured at beamline P02.2 of PETRA-III. The monochromatic incident beam with an X-ray wavelength of 0.2902 $\AA$ was focused down to approximately $3 \times 8 \mu \mathrm{m}^{2}$. The diffraction data were collected by a Perkin Elmer XRD1621 detector with the exposure time set to $120 \mathrm{~s}$ at $400 \mathrm{~K}$ and $60 \mathrm{~s}$ at $673 \mathrm{~K}$, respectively. The pressure uncertainties estimated before and after collection of each diffraction pattern were found to be less than $0.35 \mathrm{GPa}$. The background was recorded using the empty chamber in the same setup at $400 \mathrm{~K}$ and $673 \mathrm{~K}$, respectively. The $\mathrm{CeO}_{2}$ standard was used to calibrate the sample-to-detector distance and the geometrical parameters of the detector. The obtained two-dimensional (2D) images were integrated using the FIT2D software, ${ }^{21}$ and the resulting one-dimensional (1D) files in liquid regions were further converted into structure factors $S(q)$ using PDFGETX2. ${ }^{22}$ Crystalline structures were indexed using the program TREOR. ${ }^{23}$

\section{B. Molecular dynamics simulations}

The AIMD simulations of the $\mathrm{Ga}_{86} \mathrm{In}_{14}$ alloy based on the density functional theory (DFT) were accomplished by the Vienna $a b$ initio simulation package (VASP). ${ }^{24}$ The projector augmented waves (PAWs) method and the generalized gradient approximations (GGAs) of Perdew and Wang (PW91) were utilized to describe the electronic exchange and correlation potential. ${ }^{25,26} \mathrm{~A}$ canonical NVT (constant number, volume and temperature) (a)
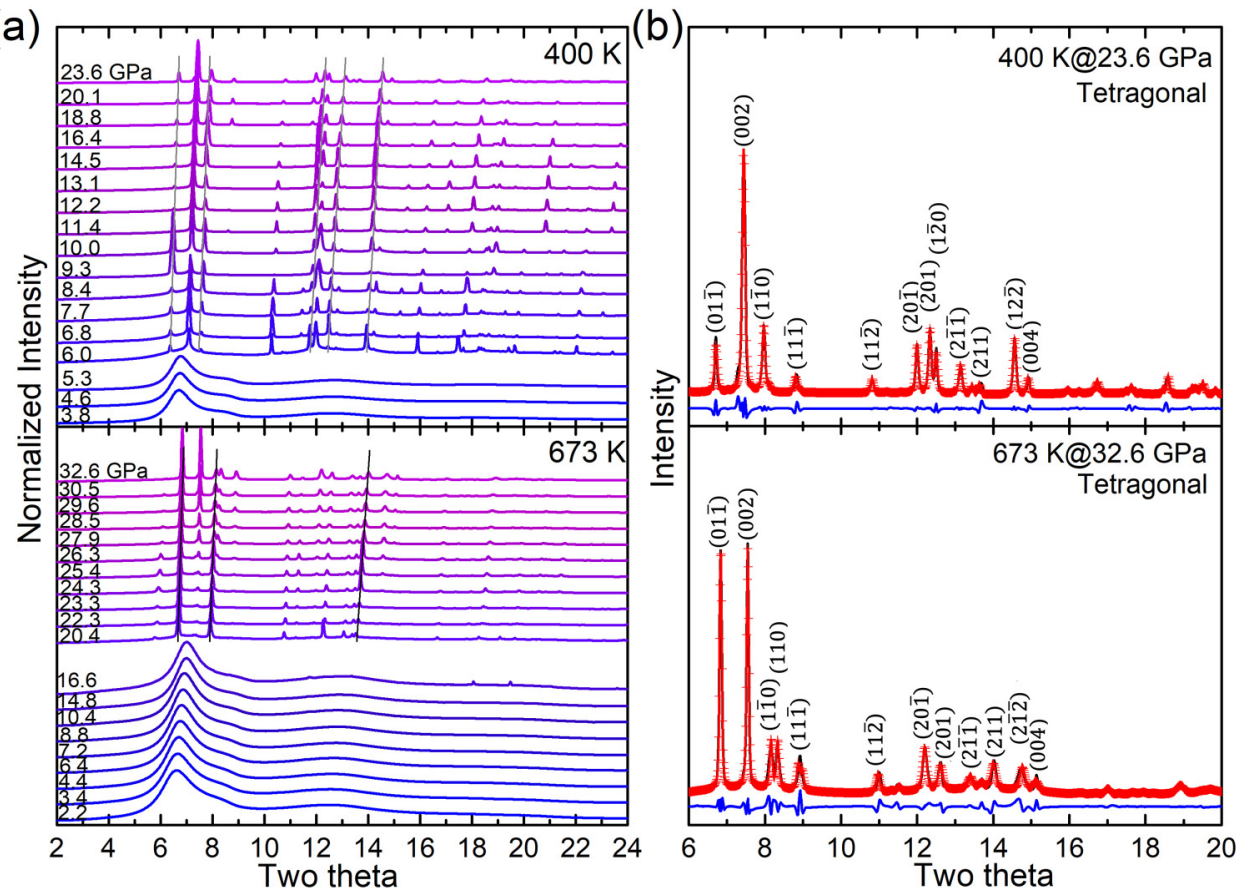

FIG. 1. (a) In situ X-ray diffraction patterns of the $\mathrm{Ga}_{85.8} \mathrm{In}_{14.2}$ eutectic alloy during compression at $400 \mathrm{~K}$ and $673 \mathrm{~K}$. (b) Integrated diffraction profiles (black lines) from the $\mathrm{Ga}_{85.8} \mathrm{In}_{14.2}$ alloy at $400 \mathrm{~K}, 23.6 \mathrm{GPa}$ and $673 \mathrm{~K}, 32.6$ $\mathrm{GPa}$ as well as the Fullprof refinement fits (red crosses). The ( $h k l)$ indices are indicated on the diffraction patterns. Below the profiles are the differences (blue lines) between the experimental and calculated data. 
ensemble was adopted, and the Nosé-Hoover thermostat ${ }^{27}$ was used to control the temperature. Newton's equation of motion was simulated via the velocity Verlet algorithm with a typical time step of $3 \mathrm{fs}$. Only the $\Gamma$ point was used to sample the Brillouin zone. The cubic box with periodic boundary conditions, containing 250 atoms (215 Ga and $35 \mathrm{In}$ ), was first thermally equilibrated at $1500 \mathrm{~K}$ for more than $18 \mathrm{ps}$ (6000 steps) to remove the memory effect and then quenched stepwise to the desired temperatures with a cooling rate of $3.33 \times 10^{13} \mathrm{~K} / \mathrm{s}$. At each temperature, the system was equilibrated by adjusting the size of the simulation box to keep the internal pressure close to zero and relaxed for more than $36 \mathrm{ps}$. More details about the simulation procedure can be found in our previous work. ${ }^{17}$ At the studied temperatures 350, 400, 500, 600, 650,673 , and $700 \mathrm{~K}$, the isothermal compression was applied by reducing stepwise the volume of the supercell with an increment of $\sim 1.3 \mathrm{GPa}$. The calculated pressure fluctuation is about $0.5 \mathrm{GPa}$. Under each pressure, the system was equilibrated for more than $36 \mathrm{ps}$ and the last $12 \mathrm{ps}$ were collected for statistical analyses.

\section{RESULTS AND DISCUSSION}

Figure 1 (a) shows the selected XRD patterns recorded during compression up to $23.6 \mathrm{GPa}$ at $400 \mathrm{~K}$ and $32.6 \mathrm{GPa}$ at $673 \mathrm{~K}$. The liquid GaIn alloy crystallizes at about $6.0 \mathrm{GPa}$ at $400 \mathrm{~K}$ and $20.4 \mathrm{GPa}$ at $673 \mathrm{~K}$, respectively. Above the crystallization pressures, the low-pressure crystalline phases at $400 \mathrm{~K}$ and $673 \mathrm{~K}$ both remain stable up to the maximum pressures achieved here. The diffraction intensities during isothermal compression are variable, especially at $400 \mathrm{~K}$, perhaps attributed to the random rotation of few crystals formed during compression. Even though it is impossible to determine the exact sites of $\mathrm{Ga}$ and In atoms in the lattice, Fullprof-type refinements still give some useful information about the crystalline structure, e.g., lattice parameter, volume of unit cell, and atomic arrangement form. Figure 1(b) shows the results of Fullprof-type refinements for the phases at $23.6 \mathrm{GPa} / 400 \mathrm{~K}$ and $32.6 \mathrm{GPa} / 673 \mathrm{~K}$. The two XRD patterns fit well with a slightly distorted I-centered tetragonal structure, which can also be expressed as a weighted average of the bct-Ga III ${ }^{28}$ and the bct-In ${ }^{29}$ structures. The deviation from the ideal tetragonal structure might be caused by several factors, e.g., the inclusion and ordering of In atoms, defects like stacking faults, or just the limited number of crystallites leading to skew integration peaks. The detailed lattice parameters and volume of unit cell at different pressures can be found in the supplementary material. The lattice parameters $(a$ or $b, c)$ or cell volumes at $400 \mathrm{~K}$ and $673 \mathrm{~K}$ vary smoothly with increasing pressure as shown in Fig. S1 in the supplementary material, again confirming the absence of polymorphism of the formed crystalline phase over the studied pressure ranges.

Since this eutectic liquid exhibits relative random and nonuniform atomic packing above and below the temperature range $400-550 \mathrm{~K},{ }^{17}$ it is necessary to investigate the pressure-induced structural evolution prior to crystallization and whether the liquid-toliquid crossover occurs in extreme conditions. Figures 2(a) and 2(b) show the full set of structure factors $S(q)$ of the eutectic liquid at $400 \mathrm{~K}$ and $673 \mathrm{~K}$ prior to crystallization, respectively. As the pressure increases, the first peak shifts to higher $q$ value, corresponding to the pressure-induced densification. The inverse of the first sharp
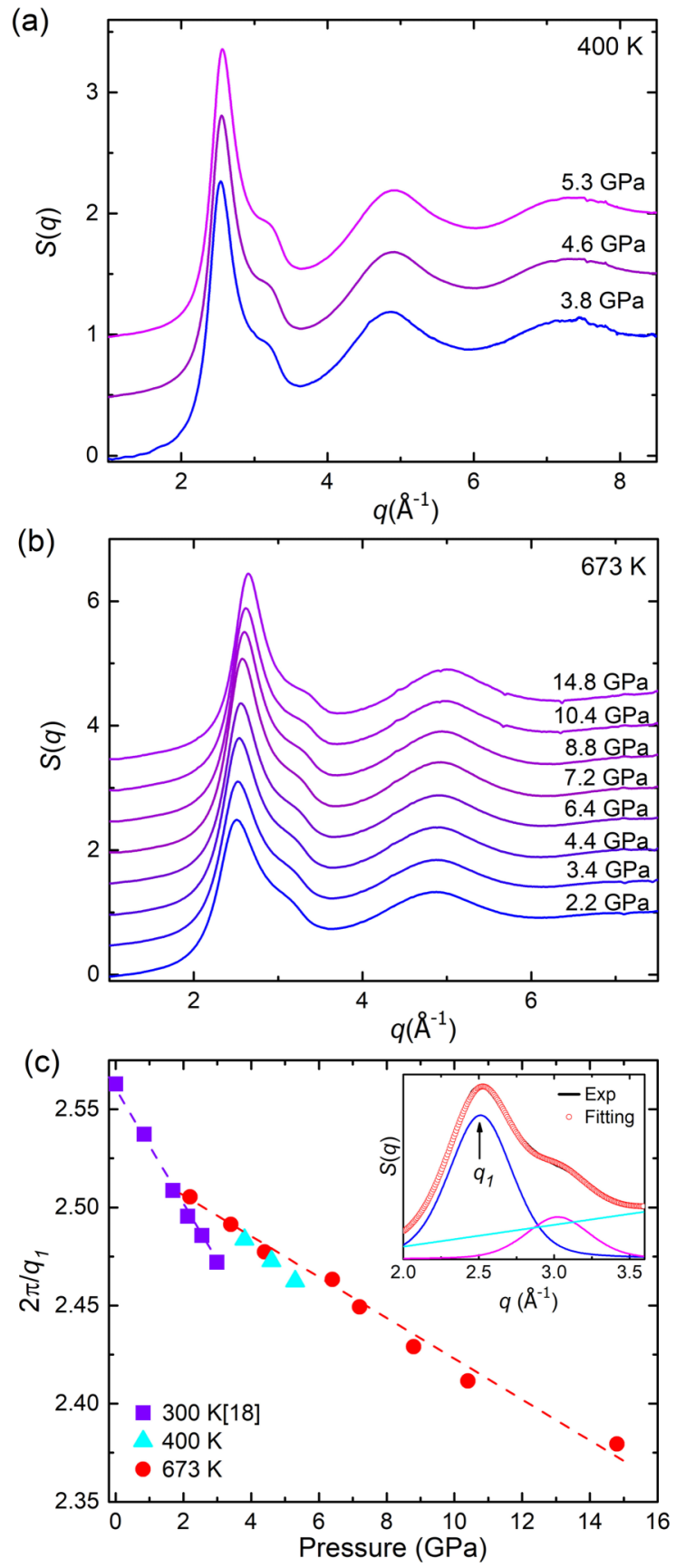

FIG. 2. Experimental $S(q)$ prior to crystallization at various pressures at (a) $400 \mathrm{~K}$ and (b) $673 \mathrm{~K}$, and (c) the corresponding inverse FSDP position with pressure. The cyan triangles and the red circles are the data at $400 \mathrm{~K}$ and $673 \mathrm{~K}$, and the data of $300 \mathrm{~K}$ (violet squares) from Ref. 18 are plotted for comparison. The dotted lines are the corresponding linear fits. Inset of (c) shows the deconvolution of the first peak in $S(q)$ using two Gaussian profiles. The black solid line represents the XRD diffraction data and red open circles for the total fitting result. The blue, magenta, and cyan curves are the two Gaussian peaks and baseline, respectively. 
diffraction peak (FSDP) position parameter $d_{\max }=2 \pi / q_{1}$ at $400 \mathrm{~K}$ and $673 \mathrm{~K}$ together with that at $300 \mathrm{~K}^{18}$ is plotted in Fig. 2(c), where $q_{1}$ is the position of first sharp diffraction peak in $S(q)$ derived using two Gaussian functions as plotted in the inset of Fig. 2(c). With increasing pressure, almost linear decreases of $d_{\max }$ indicate that no sudden structural change exists in liquid states prior to crystallization at three temperatures.

To obtain more information on the atomic-scale structural evolution and the subsequent solidification process under high $P-T$ conditions, we carried out AIMD simulations for $\mathrm{Ga}_{86} \mathrm{In}_{14}$ alloys at $350,400,500,600,650,673$, and $700 \mathrm{~K}$. Figure 3 provides a series of theoretical $g(r)$ upon isothermal compression at six different temperatures. Since the results at $650 \mathrm{~K}$ are nearly the same with those at $673 \mathrm{~K}$, we just show the detailed analysis of the latter. In the initial liquid states, the shape of all $g(r)$ curves looks similar. With increasing pressure under different temperatures, the peak heights increase, and several small peaks appear on $g(r)$ curves, indicating the transition from disordered liquid to ordered
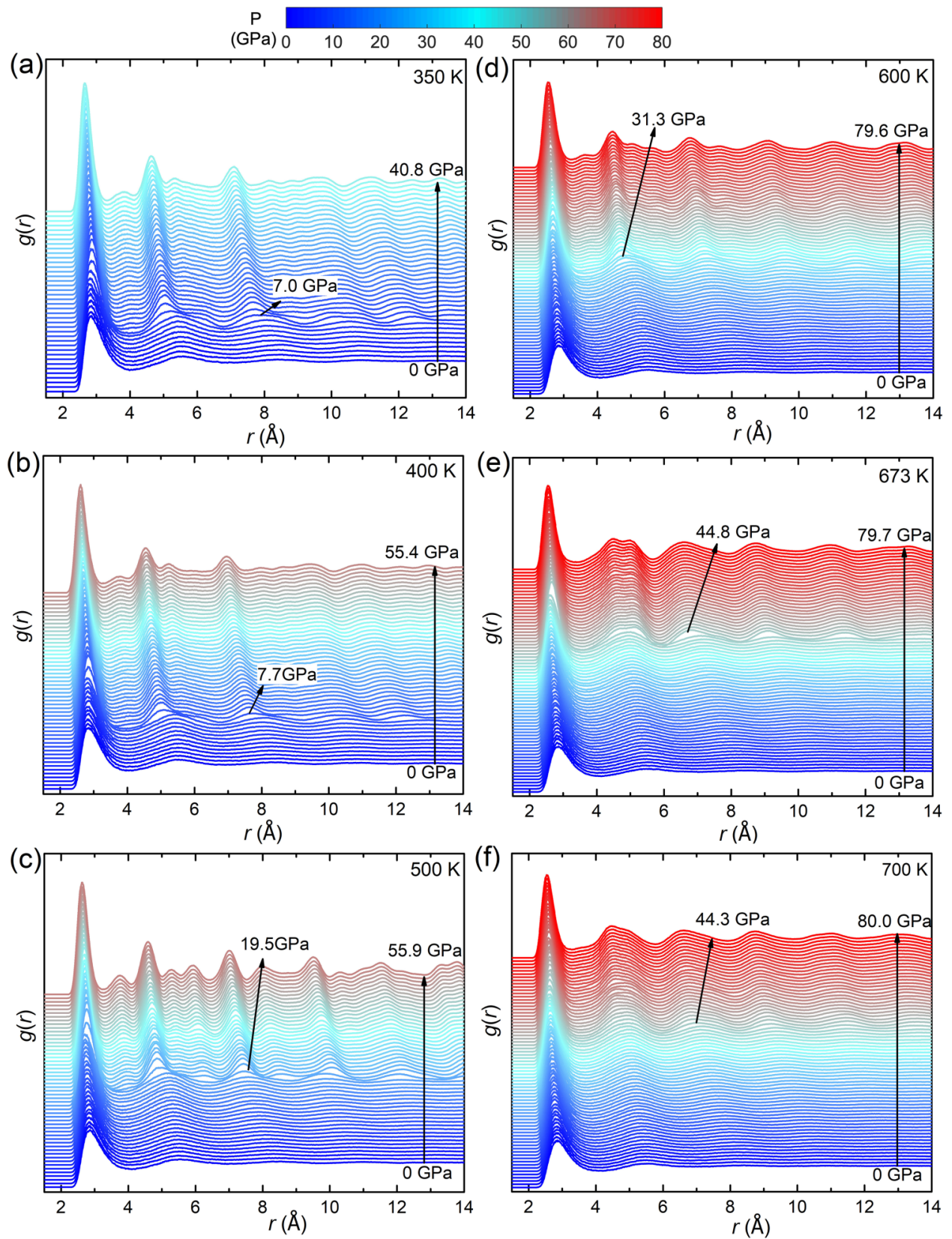

FIG. 3. A full set of theoretical $g(r)$ of the $\mathrm{Ga}_{86} \mathrm{I}_{14}$ alloy during stepwise compression along (a) 350, (b) 400, (c) 500 , (d) 600 , (e) 673 , and (f) $700 \mathrm{~K}$ isotherms. The patterns corresponding to the onset crystallization pressures at six isothermal temperatures are labeled. 
solid. The higher the isothermal temperature, the higher the transition pressure, e.g., $7.0 \mathrm{GPa}$ at $350 \mathrm{~K}, 7.9 \mathrm{GPa}$ at $400 \mathrm{~K}, 19.5 \mathrm{GPa}$ at $500 \mathrm{~K}, 31.3 \mathrm{GPa}$ at $600 \mathrm{~K}, 44.8 \mathrm{GPa}$ at $650 \mathrm{~K}$, and $44.3 \mathrm{GPa}$ at $700 \mathrm{~K}$. Before and after the transition, the main peak of $g(r)$ shifts toward shorter $r$ values upon compression due to the densification under pressure. The first peak position of $g(r)$ is usually used to evaluate the average bond length between atoms. Figure 4 shows the change in bond length, which varies almost the same with pressure along different isotherms. Only one clear jump is detected at each transition pressure where the bond length is significantly elongated. Before and after the transition point, the bond length decreases continuously with increasing pressure, suggesting that neither pressure-induced sudden structural change in the liquid nor order-to-order transition in the solid phase happens. In addition, by carefully inspecting $g(r)$ profiles of the solid phase in Fig. 3, it is interesting to find that the shapes of $g(r)$ at the final pressures under low isothermal temperatures $(350,400,500$, and $600 \mathrm{~K}$ ) are different from those under high temperatures (673 and $700 \mathrm{~K}$ ). In terms of the peak positions of $g(r)$, the former patterns display some characteristics of face centered cubic (fcc) or hcp structures while the latter patterns are close to bcc symmetry, implying that the pressure-induced solidification behaviors of the liquid GaIn eutectic alloy are essentially different when held at lowand high-temperature regions.

To further understand the pressure-induced solidification behaviors at different isothermal temperatures, the coarse-grained local bond-orientational order (BOO) methods ${ }^{30}$ were applied to monitor the atomic ordering change, which provide an accurate determination of the local atomic packing symmetry around each individual atom, especially the order parameters $Q_{6}$ combined with $W_{4}$ and $W_{6}$ have been used extensively to distinguish between liquid, bcc-, fcc-, and hcp-like atomic packings in the liquidto-solid phase transition. ${ }^{9,12,30-34}$ The detailed introduction of the

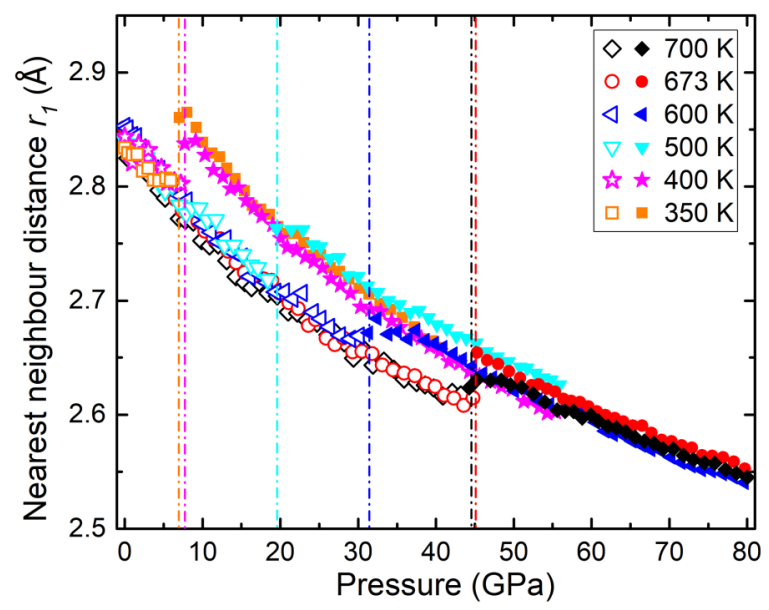

FIG. 4. Pressure-induced change of the first peak positions $r_{1}$ of the total $g(r)$ at $350,400,500,600,673$, and $700 \mathrm{~K}$. Open and solid symbols represent liquid and solid states, respectively. The vertical dashed-dotted lines show the onset crystallization pressures upon isothermal compression at six temperatures.
BOO parameters is given in the supplementary material. It should be stressed that the coarse-grained BOO parameters used here are based on the local atomic packing structures, i.e., the local symmetry does not mean that the sample must have this symmetry on the long-range scale. Therefore, in the following discussion of $\mathrm{BOO}$ analyses, we use the terms atomic packing, local order, or local symmetry to denote the atomic ordering process during the liquid-to-solid transition.

Figure 5 shows the probability distribution of $Q_{6}$ and its evolution upon compression at six different temperatures. It is clearly observed that the liquid and solid states are well separated when the threshold of $Q_{6}$ is set as 0.25 . The onset solidification pressure at each temperature determined from $Q_{6}$ well agrees with that from $g(r)$ data in Figs. 3 and 4. Prior to solidification, the $Q_{6}$ distribution is symmetric and all the values are almost less than 0.25 . When the liquid starts to solidify, the number of atoms with higher $Q_{6}$ values increases quickly and the principal peak of the $Q_{6}$ curve shifts to higher $Q_{6}$ region upon compression, indicating that highly ordered structures are formed. Below $600 \mathrm{~K}$ as shown in Figs. 5(a)-5(d), the $Q_{6}$ distribution of solidification phases covers a wide region from 0.25 to 0.6 with the principal peak at about $0.5-0.6$. However, the $Q_{6}$ above $673 \mathrm{~K}$, as plotted in Figs. 5(e) and 5(f), lies in smaller $Q_{6}$ values ranging from 0.3 to 0.5 with a symmetrical distribution at around 0.43 , again conforming a temperature dependent liquid-tosolid transition as evidenced from $g(r)$ curves in Fig. 3.

$W_{6}$ is useful to distinguish bcc-like atomic packing $\left(W_{6}>0\right)$ from close-packed orders (hcp- or fcc-like atomic packings, $W_{6}<0$ ), while $W_{4}$ is a good bond order parameter to differentiate fcc-like $\left(W_{4}<0\right)$ and hcp-like $\left(W_{4}>0\right)$ atomic packings. The nucleation of solid-like regions is initiated by high bond-orientational ordering in the liquid state ${ }^{31-33,35}$ and can be characterized by such local order parameters. Therefore, we present $W_{6}$ value for each atom and its evolution with pressure before and after solidification in Fig. 6. In liquid states, the atoms with relatively high bond order $Q_{6}(\geq 0.2)$ are picked out (red balls). Once the liquid starts to solidify, only the solid-like atoms $\left(Q_{6} \geq 0.25\right)$ are plotted (blue balls). The majority of atoms with high $Q_{6}$ have bcc-like $\left(W_{6}>0\right)$ local order in the liquid, which may act as the precursor of nucleation. As expected, with increasing applied pressure, the nucleation starts and proceeds into a bcc-like atomic packing throughout the solidification process at 673 and $700 \mathrm{~K}$, as shown in Figs. 6(e) and 6(f), respectively. However, in Figs. 6(a)-6(d), the atomic ordering transition becomes relatively complex below $600 \mathrm{~K}$. Combined with order parameters $W_{4}$, as shown in Fig. 7 , it is found that the initially formed bcc-like local order quickly converts into the fcc-like $\left(W_{6}<0\right.$ and $\left.W_{4}<0\right)$ dominated atomic packing on increasing pressure. Although the appreciable bcc-like and fcc-like local structures coexist at the early stage of nucleation at $500 \mathrm{~K}$, the fcc-like local order quickly dominates the system upon compression, implying that there exists a strong competition between fcc-like and bcc-like local orders during the liquid-to-solid transition.

To visualize the initial ordering process in the liquids, we present the spatial distribution of solid atoms $\left(Q_{6} \geq 0.25\right)$ under some representative pressures at six isothermal temperatures in Fig. 8, which clearly reveals the development of two different packing modes at the initial stage of solidification. The data at $300 \mathrm{~K}$ taken from our previous work ${ }^{18}$ are also appended for 

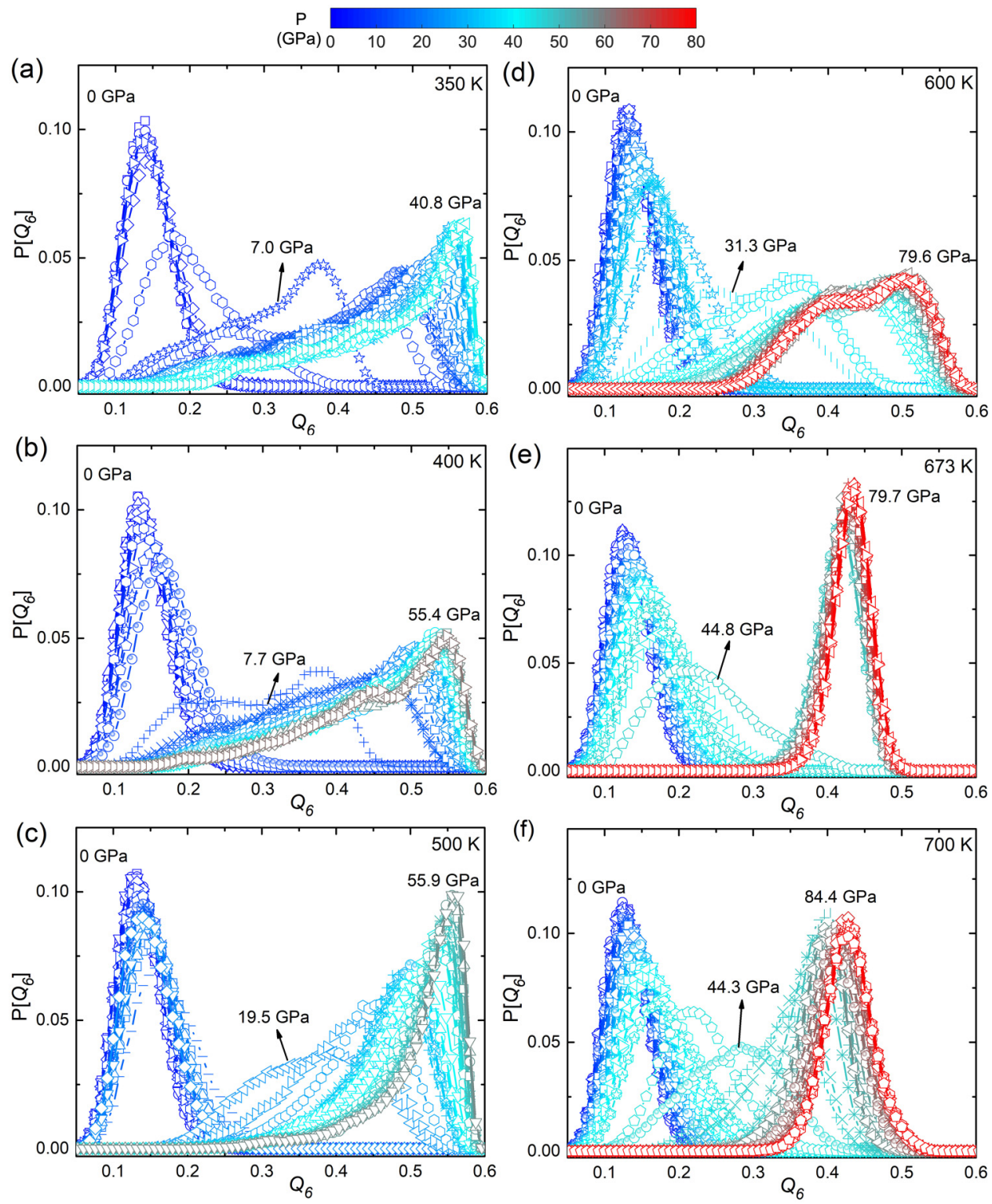

FIG. 5. Pressure-dependent probability distribution of bond-orientational order parameter $Q_{6}$ at (a) 350 , (b) 400 , (c) 500, (d) 600, (e) 673, and (f) $700 \mathrm{~K}$. comparison. It is clear that nucleation always begins with the formation of bcc-like local order at all temperatures. Further increase in pressure causes the bcc-like local order to quickly convert to the fcc-like local order and eventually develop into the fcc-like dominated atomic packing below $600 \mathrm{~K}$ while the bcc-like atomic packing remains predominant throughout the solidification process at high temperatures above $673 \mathrm{~K}$. This pressure-induced disorder-to-order transition seems in accordance with the predication of Alexander-McTague theory, ${ }^{36}$ i.e., the bcc-like nuclei should always be favored in all simple fluids.

To further validate whether the "bcc-like" atomic packing at low temperatures is in thermodynamic equilibrium or just transient intermediate, we also performed isothermal annealing at the onset crystallization pressure $(7.7 \mathrm{GPa})$ at $400 \mathrm{~K}$. As shown in Fig. S2 in the supplementary material, time-dependent energy, averaged $Q_{6}$ over all atoms, and three selected $g(r)$ at 30,150 , and 300 ps are plotted. It is found that during the relaxation of about $300 \mathrm{ps}$, the energy of the system does not change much, but the averaged $Q_{6}$ increases slightly after about 250 ps. In addition, at 300 ps, a small peak located at about $4 \AA$ emerges and the second and third peaks start to split in $g(r)$ that are the features of fcc-like atomic packing, indicating that the bcc-like atomic packing is inclined to transform into the fcc-like atomic packing during annealing. More pronounced observations are displayed in 3D simulation snapshots 

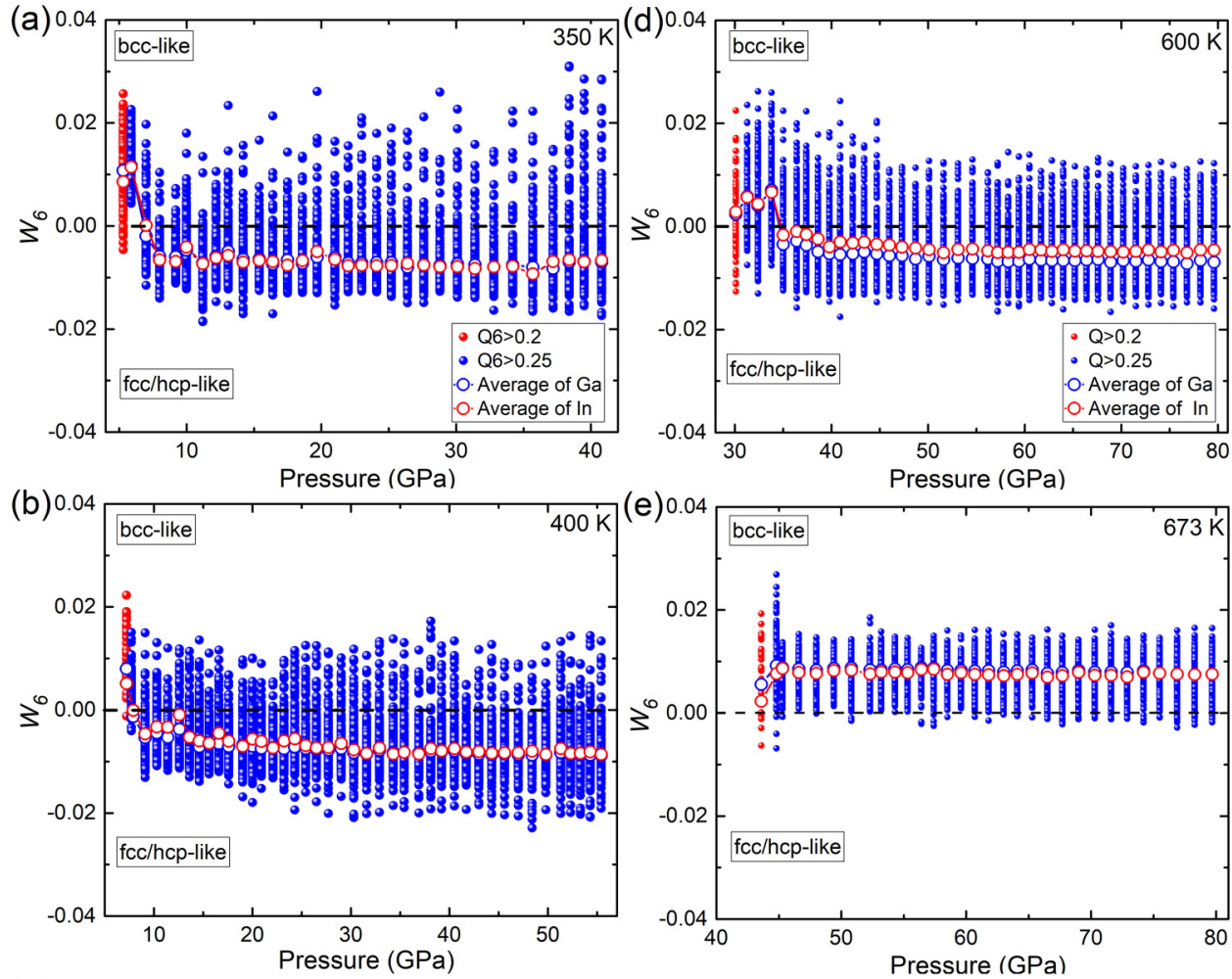

FIG. 6. The probability distribution of bond-orientational order parameter $W_{6}$ of individual element as a function of pressure together with the average value separately for $\mathrm{Ga}$ and $\mathrm{In}$ atoms before and after the nucleation process at (a) 350, (b) 400, (c) 500, (d) 600, (e) 673 , and (f) $700 \mathrm{~K}$. The red balls represent the atoms with high $Q_{6}$ $(\geq 0.2)$ in liquid states before nucleation. Blue balls are crystal-like atoms with $Q_{6} \geq 0.25$. The blue and red open circles are average $W_{6}$ values over all $\mathrm{Ga}$ and In atoms, respectively.
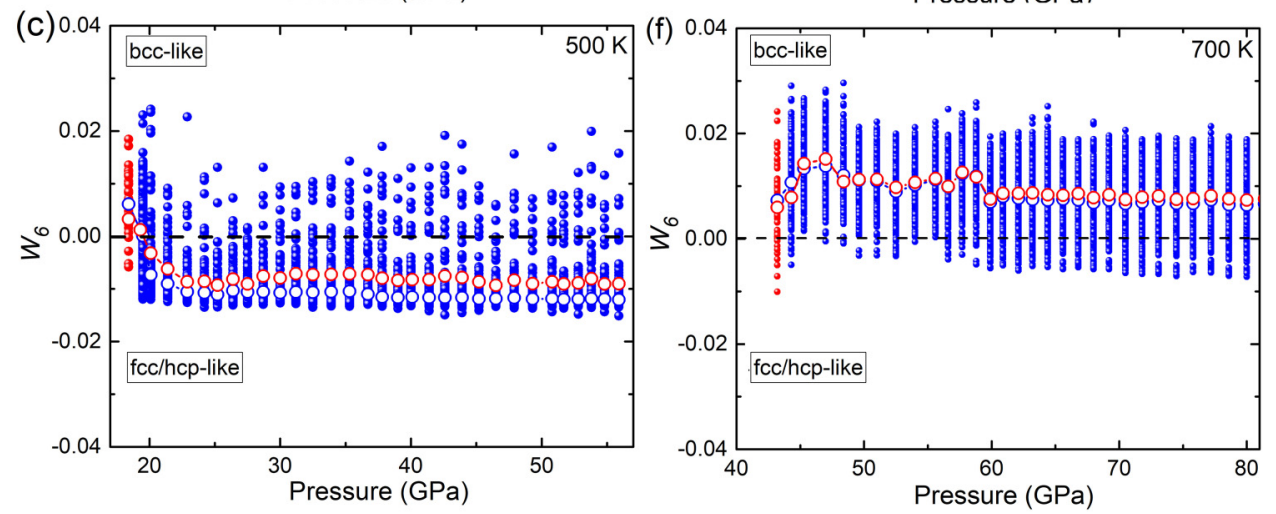

[Fig. S2(d) in the supplementary material], in which the fcc-like atomic packing becomes dominated at $300 \mathrm{ps,} \mathrm{implying} \mathrm{that} \mathrm{the}$ initially formed bcc-like atomic packing is metastable and intermediate and tends to transform into fcc-like atomic packing upon prolonged annealing.

Summarizing the theoretical and experimental results, we construct a high $P-T$ "phase diagram" of the GaIn eutectic alloy in Fig. 9, which provides a clear physical picture of liquid-to-solid transition at the atomic scale. Note that we put the term "phase diagram" in quotation marks, since we assume that the phases obtained here depend on 250 atoms system used for our AIMD simulations, regardless of the effect of small size and rapid pressure changes in simulations. Theoretical bcc- and fcc-like atomic packings are based on local order analyses. The narrow orange area enclosed by dashed lines in Fig. 9 denotes the early stage of nucleation below $650 \mathrm{~K}$ in which the bcc-like local order is initially formed, but it is in the nonequilibrium state and could be converted to the stable fcc-like atomic packing by long time annealing. Above $650 \mathrm{~K}$, the liquids form bcc-like atomic packing directly. The XRD patterns show that the liquid crystallizes into the tetragonal structures at $400 \mathrm{~K}$ and $673 \mathrm{~K}$, which can also be regarded as a slightly distorted fcc structure with local fcc-like atomic packing, consistent with the calculated low-temperature solidification structures here, but experimentally difficult to detect the transient intermediate of bcc-like atomic packing. Also, we cannot claim that the theoretically predicted high-temperature phase with bcc-like atomic 

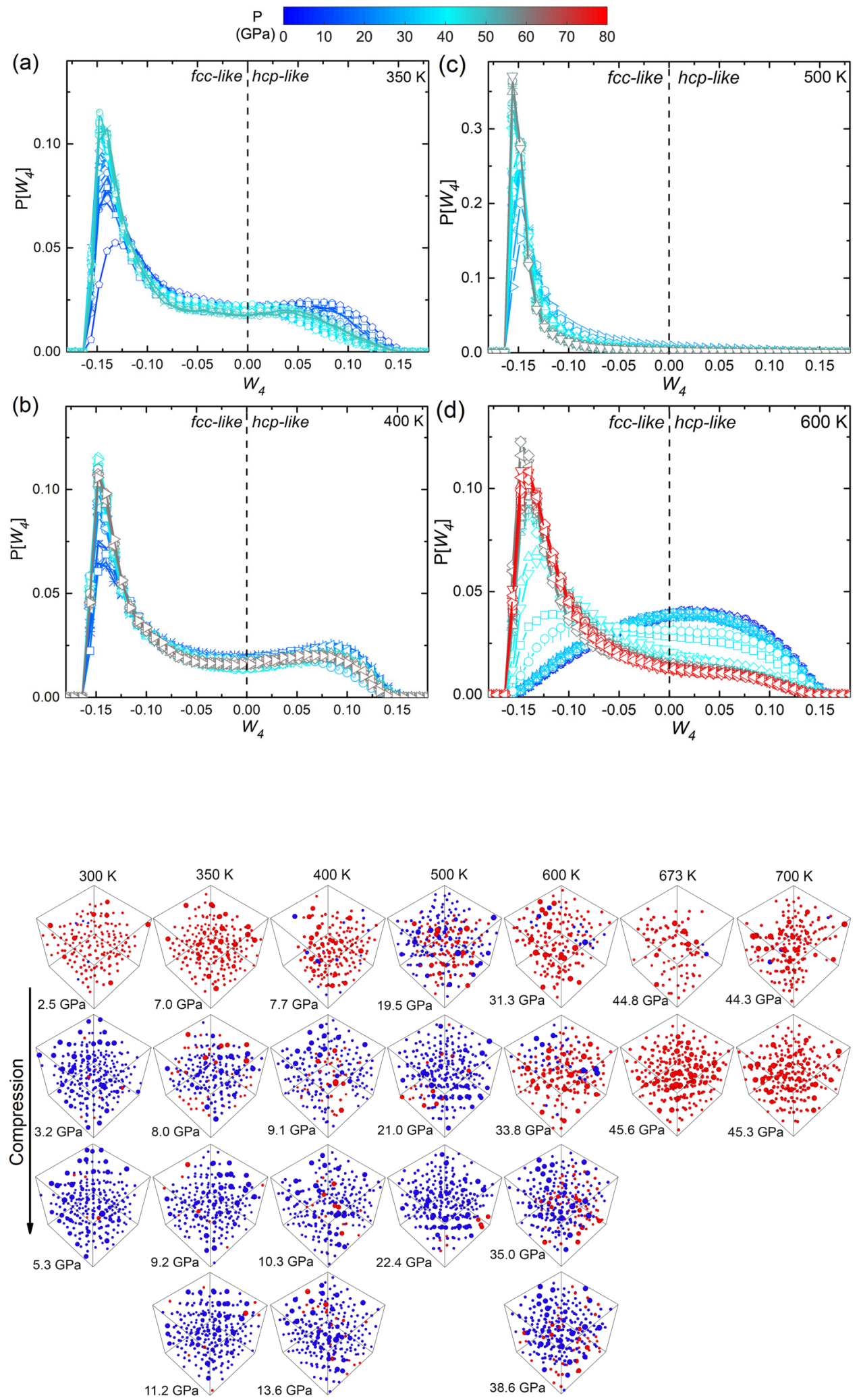

FIG. 7. The probability distribution of bond-orientational order parameter $W_{4}$ during compression after crystallization at (a) 350, (b) 400, (c) 500, and (d) $600 \mathrm{~K}$.
FIG. 8. Simulation snapshots of atomic configurations during the nucleation process at selected pressures along different isotherms. The atoms are identified according to the bondorientational order parameters. Only crystal-like atoms with $Q_{6}$ larger than 0.25 are shown. Red balls are bcc-like atoms, blue balls are fcc-like atoms, and larger size for In and smaller size for $\mathrm{Ga}$ atoms. 


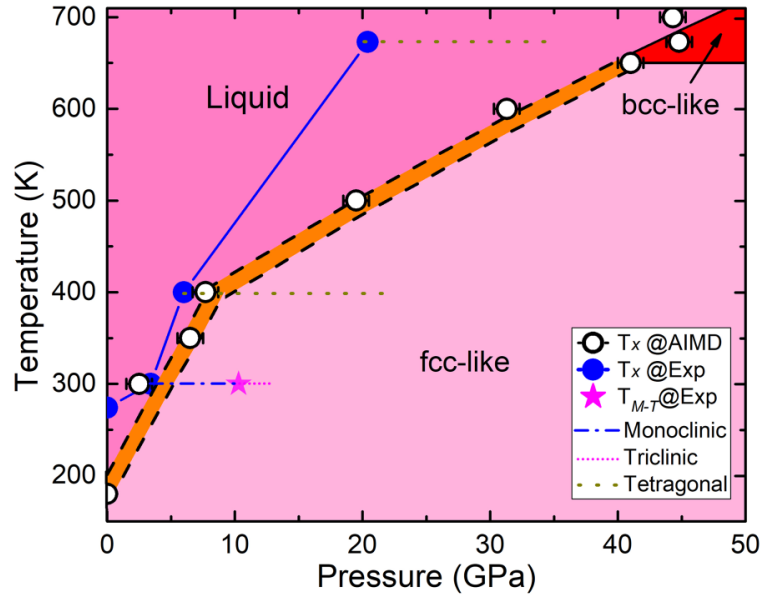

FIG. 9. "Phase diagram" derived qualitatively from AIMD simulation (open circles) and X-ray diffraction measurements (solid circles and star) in the Galn eutectic alloy. The solid lines are guides to the eye. The narrow orange region enclosed by black dashed lines below $650 \mathrm{~K}$ denotes that in this region, the nonequilibrium bcc-like local order is initially formed and tends to convert into the stable fcc-like atomic packing during long time annealing. The blue dasheddotted line, magenta short-dotted line, and dark yellow dotted line represent isothermal compression data collected over a range of pressure at 300,400 , and $673 \mathrm{~K}$, respectively, in experiments. Note that the theoretical bcc- and fcc-like atomic packings are based on local order analyses.

packing could be found in experiments upon high pressure. More studies are still needed to validate this interesting issue.

In addition, we further investigate temperature dependence of polymorph selection at fixed pressures by cooling from hightemperature melts at $673 \mathrm{~K}$ to $400 \mathrm{~K}$ and $500 \mathrm{~K}$, respectively. Figure 10 shows the evolution of pair correlation functions with time at $400 \mathrm{~K}$ and $500 \mathrm{~K}$ obtained by directly cooling from $17.9 \mathrm{GPa}$ and $29.3 \mathrm{GPa}$ at $673 \mathrm{~K}$, respectively, as well as those taken by isothermal compression of $17.6 \mathrm{GPa}$ at $400 \mathrm{~K}$, and $28.7 \mathrm{GPa}$ at $500 \mathrm{~K}$. The insets show the temporal evolution of total potential energy and the development of local atomic packing ordering $\left(Q_{6} \geq 0.25\right)$ with time after cooling. It is clear that two stages can be differentiated based on the potential energy of systems, i.e., the liquid state with higher energy and solid regions with lower energy. In order to clearly illustrate the time dependence of the atomic ordering, we selected three representative times $(20,100$, and $300 \mathrm{ps})$ labeled as A, B, and C, respectively, to represent how atomic packing changes before, during, and after the nucleation process upon isothermal annealing. It is observed that after cooling to 400 and $500 \mathrm{~K}$, the high-temperature liquid structure still remained after 20 ps of isothermal annealing at $500 \mathrm{~K}$, as shown in $g(r)$ and atomic configuration denoted by $\mathrm{A}$ in Fig. 10. With increasing annealing time to about $100 \mathrm{ps}$, the bcc-like local order is detected as marked by B. Over time, the bcc-like atomic packing gradually converts into the fcc-like form, which becomes dominant eventually after $300 \mathrm{ps}$ in calculations as labeled by C. Ultimately, similar local structures are also obtained from the isothermal compression at $400 \mathrm{~K}$ and $500 \mathrm{~K}$ under approximate pressure conditions, validating the phase diagram in Fig. 9.
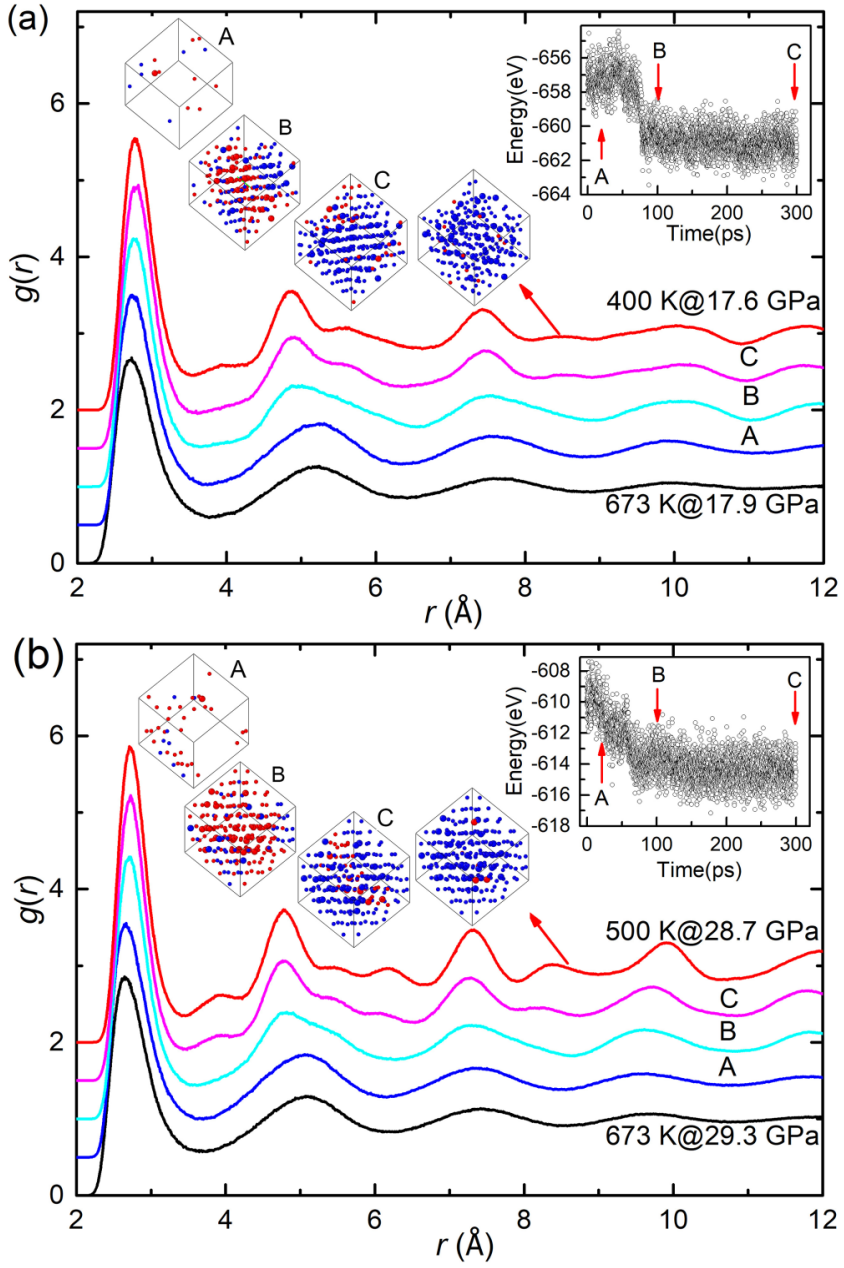

FIG. 10. The theoretical $g(r)$ and its evolution with time (blue, cyan, and pink lines) at (a) 400 and (b) $500 \mathrm{~K}$ obtained by directly cooling from hightemperature melts at $673 \mathrm{~K}$ at $17.9 \mathrm{GPa}$ (black line) and $29.3 \mathrm{GPa}$ (black line), respectively. The red lines are derived from isothermal compression at (a) $400 \mathrm{~K}$ and $17.6 \mathrm{GPa}$ and (b) $500 \mathrm{~K}$ and $28.7 \mathrm{GPa}$. The insets in the upper right corner of (a) and (b) show the temporal evolution of total potential energy isothermally annealed at $400 \mathrm{~K}$ and $500 \mathrm{~K}$ after cooling from $673 \mathrm{~K}$, respectively. Three representative annealing times $(20,100$, and $300 \mathrm{ps})$ are selected, labeled as A, B, and $C$, respectively, representing the changes in atomic packing before, during, and after the nucleation process. Their corresponding pressure values are 17.6 $\mathrm{GPa}(\mathrm{A}), 17.4 \mathrm{GPa}(\mathrm{B})$, and $17.4 \mathrm{GPa}(\mathrm{C})$ at $400 \mathrm{~K}$ in (a) and $29.0 \mathrm{GPa}(\mathrm{A})$, $28.8 \mathrm{GPa}(\mathrm{B})$, and $28.8 \mathrm{GPa}(\mathrm{C})$ at $500 \mathrm{~K}$ in (b). The 3D snapshots of atomic configurations at three times as well as those from isothermal compression at $400 \mathrm{~K} / 17.6 \mathrm{GPa}$ and $500 \mathrm{~K} / 28.7 \mathrm{GPa}$ are also given. Note that only crystal-like atoms are shown, in which red balls are bcc-like atoms and blue balls are fcc-like atoms.

\section{CONCLUSIONS}

In summary, we investigate the structural evolution in the liquid GaIn eutectic alloy and subsequent liquid-to-solid transition under high $P-T$ conditions using X-ray diffraction combined with 
$a b$ initio molecular dynamics simulations. As a result, no sudden structural changes are detected in the liquid states during isothermal compressions but only pressure-induced disorder-to-order transition during solidification. The experimental results reveal that the liquids at 400 and $673 \mathrm{~K}$ are both solidified into tetragonal crystalline phases with fcc-type local atomic packings. The theoretical simulations successfully describe the disorder (liquid)-to-order (solid) transition at the atomic scale and reveal different liquid-to-solid transition behaviors at high and low temperatures. Above $650 \mathrm{~K}$, the bcc-like atomic packing is always predominant during solidification and stays stable over the pressure range investigated. However, below $650 \mathrm{~K}$, a number of intermediate metastable bcc-like atomic packings are initially formed at the early stage of nucleation but quickly convert into the fcc-like atomic packing with increasing applied pressure, whose structure is locally similar to the solidification phases in experiments. Based on the experimental and theoretical results, a high-temperature and high-pressure "phase diagram" of the GaIn eutectic alloy is established, which could give a clear picture for solidification behavior at the atomic scale and also open up the possibilities for controlling the solidification structures by tuning the external conditions.

\section{SUPPLEMENTARY MATERIAL}

See the supplementary material for the detailed lattice parameters and volume of unit cell at different pressures taken from Fullprof-type refinements of the GaIn eutectic alloy in experiments as well as the explicit introduction of $\mathrm{BOO}$ parameters and isothermal annealing data under $7.7 \mathrm{GPa}$ at $400 \mathrm{~K}$ in simulations.

\section{ACKNOWLEDGMENTS}

Financial support from the National Natural Science Foundation of China (NNSFC) (Nos. U1832203, 51671169, 51671170, and U1532115), the National Key Research and Development Program of China (Nos. 2016YFB0700201, 2016YFB0701203, and 2017YFA0403404), the NSF of Zhejiang Province (Nos. Z1110196, Y4110192, and LY15E010003), and the Fundamental Research Funds for the Central Universities is gratefully acknowledged. The computer resources at National Supercomputer Centers in Tianjin and Special Program for Applied Research on Super Computation of the NSFC-Guangdong Joint Fund (the second phase) under Grant No. U1501501 are also gratefully acknowledged. The beamline support at P02.2 of PETRA-III is also greatly appreciated.

\section{REFERENCES}

${ }^{1}$ K. F. Kelton and A. L. Greer, Nucleation in Condensed Matter: Application in Materials and Biology (Elsevier, Amsterdam, 2010).

${ }^{2}$ X.-D. Wang and J.-Z. Jiang, Adv. Mater. 29, 1703136 (2017).

${ }^{3}$ S. Wei, F. Yang, J. Bednarcik, I. Kaban, O. Shuleshova, A. Meyer, and R. Busch, Nat. Commun. 4, 2083 (2013).
${ }^{4}$ A. Cadien, Q. Y. Hu, Y. Meng, Y. Q. Cheng, M. W. Chen, J. F. Shu, H. K. Mao, and H. W. Sheng, Phys. Rev. Lett. 110, 125503 (2013).

${ }^{5}$ L. Zhong, J. Wang, H. Sheng, Z. Zhang, and S. X. Mao, Nature 512, 177-180 (2014).

${ }^{6}$ W. Xu, M. T. Sandor, Y. Yu, H.-B. Ke, H.-P. Zhang, M.-Z. Li, W.-H. Wang, L. Liu, and Y. Wu, Nat. Commun. 6, 7696 (2015).

${ }^{7}$ Y. Shibuta, S. Sakane, E. Miyoshi, S. Okita, T. Takaki, and M. Ohno, Nat. Commun. 8, 10 (2017).

${ }^{8}$ Y. Sun, H. Song, F. Zhang, L. Yang, Z. Ye, M. I. Mendelev, C.-Z. Wang, and K.-M. Ho, Phys. Rev. Lett. 120, 85703 (2018).

${ }^{9}$ U. Gasser, E. R. Weeks, A. Schofield, P. N. Pusey, and D. A. Weitz, Science 292, 258-262 (2001).

${ }^{10}$ T. Kawasaki and H. Tanaka, Proc. Natl. Acad. Sci. U.S.A. 107, 14036-14041 (2010).

${ }^{11}$ J. Bokeloh, R. E. Rozas, J. Horbach, and G. Wilde, Phys. Rev. Lett. 107, 145701 (2011).

${ }^{12}$ P. Tan, N. Xu, and L. Xu, Nat. Phys. 10, 73-79 (2014).

${ }^{13}$ Y. Shibuta, S. Sakane, T. Takaki, and M. Ohno, Acta Mater. 105, 328-337 (2016).

${ }^{14}$ M. D. Dickey, R. C. Chiechi, R. J. Larsen, E. A. Weiss, D. A. Weitz, and G. M. Whitesides, Adv. Funct. Mater. 18, 1097-1104 (2008).

${ }^{15}$ G. Schwartz, B. C.-K. Tee, J. Mei, A. L. Appleton, D. H. Kim, H. Wang, and Z. Bao, Nat. Commun. 4, 1859 (2013).

${ }^{16}$ Y. Gao, H. Ota, E. W. Schaler, K. Chen, A. Zhao, W. Gao, H. M. Fahad, Y. Leng, A. Zheng, F. Xiong, C. Zhang, L. Tai, P. Zhao, R. S. Fearing, and A. Javey, Adv. Mater. 29, 1701985 (2017).

${ }^{17}$ Q. Yu, X. D. Wang, Y. Su, Q. P. Cao, Y. Ren, D. X. Zhang, and J. Z. Jiang, Phys. Rev. B 95, 224203 (2017).

${ }^{18}$ Q. Yu, A. S. Ahmad, K. Ståhl, X. D. Wang, Y. Su, K. Glazyrin, H. P. Liermann, H. Franz, Q. P. Cao, D. X. Zhang, and J. Z. Jiang, Sci. Rep. 7, 1139 (2017).

${ }^{19}$ K. D. Litasov, P. N. Gavryushkin, P. I. Dorogokupets, I. S. Sharygin, A. Shatskiy, Y. Fei, S. V. Rashchenko, Y. V. Seryotkin, Y. Higo, K. Funakoshi, and E. Ohtani, J. Appl. Phys. 113, 133505 (2013).

${ }^{20}$ S. V. Popova and L. N. Fomicheva, J. Less Common Metals 77, 137-140 (1981).

${ }^{21}$ A. P. Hammersley, S. O. Svensson, M. Hanfland, A. N. Fitch, and D. Hausermann, High Pressure Res. 14, 235-248 (1996).

${ }^{22}$ X. Qiu, J. W. Thompson, and S. J. L. Billinge, J. Appl. Crystallogr. 37, 678 (2004).

${ }^{23}$ P. E. Werner, L. Eriksson, and M. Westdahl, J. Appl. Cryst. 18, 367-370 (1985).

${ }^{24}$ W. G. Hoover, Phys. Rev. A 31, 1695-1697 (1985).

${ }^{25}$ G. Kresse and J. Furthmuller, Phys. Rev. B 54, 11169-11186 (1996).

${ }^{26}$ P. E. Blöchl, Phys. Rev. B 50, 17953-17979 (1994).

${ }^{27}$ S. Nosé, J. Chem. Phys. 81, 511-519 (1984).

${ }^{28}$ L. Bosio, J. Chem. Phys. 68, 1221-1223 (1978).

${ }^{29}$ C. Kittel, Introduction to Solid State Physics (Wiley, New York, 1996).

${ }^{30}$ W. Lechner and C. Dellago, J. Chem. Phys. 129, 114707 (2008).

${ }^{31}$ T. T. Debela, X. D. Wang, Q. P. Cao, D. X. Zhang, J.-J. Zhu, and J. Z. Jiang, J. Appl. Phys. 117, 114905 (2015).

${ }^{32}$ T. T. Debela, X. D. Wang, Q. P. Cao, Y. H. Lu, D. X. Zhang, H.-J. Fecht, H. Tanaka, and J. Z. Jiang, Phys. Rev. B 89, 104205 (2014).

${ }^{33}$ J. Russo and H. Tanaka, Sci. Rep. 2, 505 (2012).

${ }^{34}$ S. A. Khan, X. D. Wang, Q. P. Cao, D. X. Zhang, and J. Z. Jiang, J. Appl. Phys. 124, 225903 (2018)

${ }^{35}$ H. Tanaka, Eur. Phys. J. E 35, 113 (2012).

${ }^{36}$ S. Alexander and J. McTague, Phys. Rev. Lett. 41, 702-705 (1978). 